\title{
EMERGING INFECTIOUS DISEASES: COPING WITH UNCERTAINTY
}




\begin{abstract}
The world's scientific community must be in a state of constant readiness to address the threat posed by newly emerging infectious diseases. Whether the disease in question is SARS in humans or BSE in animals, scientists must be able to put into action various disease containment measures when everything from the causative pathogen to route(s) of transmission is essentially uncertain. A robust epistemic framework, which will inform decision-making, is required under such conditions of uncertainty. I will argue that this framework should have reasoning at its centre and, specifically, that forms of reasoning beyond deduction and induction should be countenanced by scientists who are confronted with emerging infectious diseases. In previous articles, I have presented a case for treating certain so-called traditional informal fallacies as rationally acceptable forms of argument that can facilitate scientific inquiry when little is known about an emerging disease. In this paper, I want to extend that analysis by highlighting the unique features of these arguments that makes them specially adapted to cope with conditions of uncertainty. Of course, such a view of the informal fallacies must at least be consistent with the reasoning practices of scientists, and particularly those scientists (viz. epidemiologists) whose task it is to track and respond to newly emerging infectious diseases. To this end, I draw upon examples of scientific reasoning from the UK's BSE crisis, a crisis that posed a significant threat to both human and animal health.
\end{abstract}

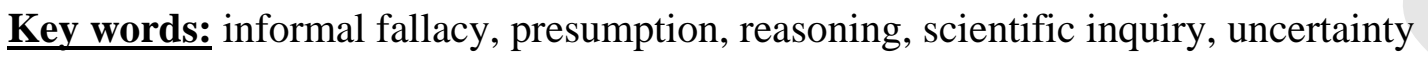




\section{EMERGING INFECTIOUS DISEASE AND UNCERTAINTY}

Emerging infectious diseases present public health scientists with a uniquely difficult challenge. These diseases pose a potentially serious threat to the health of both human and animal populations, a threat that demands action from these scientists. Yet, this action must often be undertaken in conditions that are characterized by a pervasive lack of knowledge. Recent human and animal epidemics provide clear examples of the uncertainty and ignorance that attend the emergence of new diseases. In 2000, an inquiry into the UK's BSE crisis ${ }^{1}$ revealed something of the extent of this uncertainty and lack of knowledge. Mr Cruickshank of the Animal Health Group in the UK's Ministry of Agriculture, Fisheries and Food remarked that '[w]e do not know where this disease came from, we do not know how it is spread and we do not know whether it can be passed to humans' (BSE Inquiry Report, Volume 1: 44). Even more recently, global scientific uncertainty has been strikingly evident in the case of the emergence of severe acute respiratory syndrome (SARS). Lingappa et al. (2004) stated that in March 2003, when the global health community first became aware of the emergence of SARS:

[T]he international health community faced a potential pandemic for which there were no identified causal agent, no diagnostic laboratory assays, no defined properties or risk factors for transmission, no infection-control practices of proven efficacy, and no known treatment or prevention measures (167). 
The BSE and SARS epidemics were eventually contained. ${ }^{2}$ However, these outbreaks of infectious disease, and others like them, have occasioned reflection on the part of investigators about how uncertainty may best be tackled in future epidemics. ${ }^{3}$ Nowhere is the need for such reflection more pronounced than in epidemiology, the branch of science that is responsible for detecting and monitoring the emergence of new infectious diseases. While mathematical modelling of epidemics is commonplace in epidemiology, the modes of reasoning that are integral to these models are construed along strictly deductiveinductive lines. ${ }^{4}$ A reorientation away from deductive and inductive modes of reasoning towards presumptive frameworks is central to the view of scientific reasoning that I will discuss in this paper (and may even assist scientists as they engage in reflection of how epidemiology may best respond to uncertainty $\left.{ }^{5}\right)$. Specifically, I will argue that the presumptive character of certain informal fallacies makes them ideally suited to the task of advancing scientific inquiry in contexts of uncertainty. It is to an examination of these fallacies and their role in scientific reasoning that we now turn.

\section{INFORMAL FALLACIES AND SCIENTIFIC INQUIRY}

The history of the informal fallacies has been an interesting one to say the least. Since Aristotle first examined the fallacies in Sophistical Refutations, and with the exception of contributions from medieval logicians and theorists such as Sidgwick, Whately and Mill, the fallacies languished in a state of almost total neglect until the latter part of the twentieth century. In 1970, Charles Hamblin's book Fallacies reversed the fortune of this branch of logical inquiry in a way that is still being felt to the present day. Specifically, Hamblin was concerned to challenge the so-called standard treatment of the fallacies that 
was found in logic textbooks. ${ }^{6}$ His own analysis of the fallacies emphasized the use of dialectical structures for the normative evaluation of arguments, a point that had been largely overlooked in the study of fallacies but which had been central to Aristotle's original account. Hamblin's dialectical framework was significant in the following respect. It spawned a number of studies that were to describe how variants of the informal fallacies were nonfallacious in particular contexts of use. In a study of nonfallacious arguments from ignorance, for example, Walton (1992) directly attributes the presumptive framework that is the basis of his analysis to Hamblin's formal dialectic:

This analysis of presumption clearly itself presumes the existence of a dialectical framework for the evaluation of arguments where two parties "reason together". But such a framework is given in the outline of formal dialectic presented by Hamblin (1970)... (Walton 1992: 383).

Steadily, one informal fallacy after another was shown to have nonfallacious variants. By far the most significant contributors to this area of fallacy theory have been John Woods and Douglas Walton. In a large number of books and journal articles, these theorists described nonfallacious forms of petitio principii (begging the question), argumentum ad ignorantiam (the argument from ignorance), and argumentum ad baculum (the argument from the stick or appeal to force), amongst others (Walton 1985, 1992; Woods 1995). Informal fallacies were now experiencing an intellectual renaissance that had not been witnessed in many earlier centuries of inquiry in logic. As more and more theorists turned their attention to the rational and epistemic features of the informal fallacies, it became 
clear that these arguments, and the presumptive frameworks that licensed them, had applications in domains where deductive and inductive modes of reasoning had previously dominated. One such domain was scientific inquiry. In this way, in Cummings (2000, 2002, 2004) I described how nonfallacious forms of three informal fallacies petitio principii, arguments from analogy and argumentum ad ignorantiam - functioned as reasoning heuristics that facilitate the progress of scientific inquiry under conditions of epistemic uncertainty. How surprising this new found status of the fallacies would have appeared to the logicians of earlier centuries. ${ }^{7}$

Some consideration of informal fallacies and presumptive frameworks in general reveals why these arguments are well suited to the analysis of reasoning within scientific contexts. Presumption is a rather tentative epistemic category. It contains a commitment to a thesis that is of an altogether weaker nature than either knowledge or belief, for example. ${ }^{8}$ This commitment can be easily eroded in the presence of countervailing conditions. So an agent may presume that $p$, but relinquish commitment to $p$ as soon as conditions give the slightest indication to reject $p$. The tentative, provisional nature of presumption is described by Rescher as follows:

[A] presumption is a plausible pretender to truth whose credentials may well prove insufficient, a runner in a race it may not win. The "acceptance" of a proposition as a merely presumptive truth is not acceptance at all but a highly provisional and conditional epistemic inclination toward it, an inclination that falls far short of outright commitment (2006: 3). 
These features of presumption mean that this epistemic concept is particularly well suited to an analysis of reasoning in the context of scientific inquiry in at least three respects. Firstly, the fallible nature of scientific inquiry demands that our various epistemic concepts must be able to withstand error and failure. Moreover, the fact of the occurrence of error should not erode our confidence in those concepts which deliver truths to us on enough occasions for us to continue to operate by them. Presumption, it seems, is the ideal epistemic candidate for such a fallible process of scientific inquiry:

Their inherent defeasibility means that appropriate presumptions are impervious to occasional failure (Rescher 2006: 7).

Secondly, many scientific inquiries are conducted under conditions of extreme urgency and uncertainty. Where the health of the public is at risk, as is the case in a newly emerging infectious disease, urgency and uncertainty become uneasy bedfellows. In order to maximize the urgency of a scientific response to an epidemic, scientists are often compelled to institute disease containment measures using knowledge bases that are incomplete and uncertain. We saw in section 1, for example, how scientists investigating SARS and BSE lacked knowledge of all key aspects of the epidemics that confronted them (types of pathogen, route(s) of transmission, etc.). However, if the implementation of containment measures must await the development of certain knowledge bases, the urgency of a scientific response will be compromised with possible devastating consequences for the health of a population. Presumption is the epistemic concept that 
enables scientists to balance the practical demands of disease containment against the theoretical requirement for complete and certain knowledge. For it is presumption that licenses decisions of the practical sphere when complete and certain knowledge is beyond the immediate grasp of scientists:

[P]resumption is certainly not knowledge: we do not know what we merely presume to be so. As an informative resource its standing is quite different from that of knowledge... [presumption] nevertheless is an informative resource - and a highly useful one at that, since it serves to close up an otherwise debilitating gap (Rescher 2006: 6; italics in original).

Thirdly, in yielding to considerations that are more plausible, presumptions are inextricably linked to the standards and types of evidence that apply in a particular domain. The evidence that is required to defeat a presumption varies on the basis of context-specific considerations such as the importance of the question at issue. ${ }^{9}$ For example, one might reasonably expect scientists who are testing a new drug for use in humans to have to meet a very high burden of proof before such a drug can be licensed. In such a case, the precautionary principle ${ }^{10}$ requires that there is a presumption against the safety of the new drug which extensive clinical trials must seek to overcome. As more and more trials are conducted and no adverse health effects of the drug are observed, the presumption shifts in favour of the safety of the new drug. Long-standing presumptions grow in epistemic stature as they are 'retrospectively validated' by inquiry. ${ }^{11}$ This capacity of presumptions to improve their epistemic standing is the direct result of the 
dialectical to and fro that occurs between presumption and burden of proof in scientific inquiry. Where this dialectical exchange does not occur, or is otherwise subverted, it is usually to the detriment of scientific inquiry. We will see subsequently how a presumption in support of the non-transmissibility of BSE to humans became 'fossilized' in the BSE inquiry with quite disastrous consequences for human health.

These three features of presumption are significant in the following respect: they are integral to the view of the informal fallacies that I am espousing in this paper, a view in which these fallacies facilitate scientific inquiry under conditions of uncertainty. To demonstrate this claim, I will draw on two earlier analyses of fallacies in Cummings (2002, 2004). However, where those earlier analyses described the logical features and functions of the fallacies in question, there was little consideration of their presumptive character. That presumptive character, I will argue, is what makes the fallacies ideally suited to the rational methodology of science.

\subsection{Scientific Error and the Informal Fallacies}

The reason logicians are so quick to legislate against the fallacies is that they are standardly taken to lead us into error. ${ }^{12}$ Error is an interesting concept which has hidden dimensions that have relevance for the account of fallacies that I am developing in the present context. One of those dimensions is the persistence of error. Notwithstanding our best efforts to minimize and even eliminate errors, they are a persistent feature of our various cognitive deliberations. ${ }^{13}$ The persistence of error in the form of fallacies is remarked upon by John Woods. Woods is struck by the recurrence of certain fallacies 
(the so-called 'gang of eighteen ${ }^{14}$ ) in the literature. His explanation of their recurrence 'generation-in and generation-out' is revealing of the status that Woods affords to the fallacies:

[T]he standard examples caricature (because they over-simplify) improprieties of rational performance of which they themselves can be considered a kind of idealized symptom. Those misperformances in turn are failures of one or another of our basic rational survival skills (2004: 10-11).

Woods clearly links the persistence of the fallacies to the role they play in our cognitive rationality. I agree with this view in part - fallacies have a much closer connection to our cognitive rationality than logicians have typically acknowledged. But I think Woods tends to follow other logicians in presenting a rather negative case against the fallacies. Quite apart from being 'improprieties of rational performance', the fallacies, I contend, are a core component of our rational competence. Of course, on occasion, they let us down - we can make errors based on the particular cognitive directions in which they lead us. But these errors do little damage to the rational standing of the argument forms in question. This is because presumption is the very essence of these argument forms and, like presumption, these forms are 'impervious to occasional failure'. This is amply demonstrated by two informal fallacies - the argument from ignorance and the argument from analogy - which played a key role in the reasoning of BSE scientists. The argument from ignorance was used so extensively during the BSE crisis in the UK that the public inquiry ${ }^{15}$ that was set up to investigate the handling of the epidemic labeled it as the 'mantra' of the BSE story. Although different forms of the argument were used, it was 
most commonly employed in relation to the question of the transmissibility of BSE to humans. In this way, Dr Calman (Chief Medical Officer, 1991-1998) released a statement on 26 January 1994 in which he stated:

[O]n the basis of the work done so far, there was no evidence whatever that BSE caused CJD and, similarly, not the slightest evidence that eating beef or hamburgers caused CJD (BSE Inquiry Report, Volume 1: 143; italics added).

On 7 December 1995, Dr Robert Kendell (Chief Medical Officer for Scotland, 19911996) made a public statement in which he claimed '[w]e have no evidence of any connection between BSE and CJD' (BSE Inquiry Report, Volume 1: 150; italics added). In a letter to the Chief Medical Officer dated 24 July 1990, the Spongiform Encephalopathy Advisory Committee (SEAC) ${ }^{16}$ concluded that:

[W]e believe that there is no scientific justification for not eating British beef and that it can be eaten by everyone (BSE Inquiry Report, Volume 1: 131; italics added).

These repeated claims by scientists ${ }^{17}$ that there was 'no evidence' that BSE caused CJD in humans and that there was 'no scientific justification' for not eating British beef had the effect of persuading an anxious public that BSE was not transmissible to humans. The vehicle of this persuasion was a classic argument from ignorance ${ }^{18}$ which can be reconstructed as follows: 
There is no evidence that BSE in cattle causes CJD in humans.

$\therefore$ BSE in cattle does not cause CJD in humans.

That BSE would not transmit to humans was also the conclusion of a second argument form that was used extensively by BSE scientists. This was the argument from analogy, where scientists used purported similarities of BSE with scrapie disease in sheep to argue that BSE would not transmit to humans. Analogical reasoning was integral to the deliberations of the Southwood Working Party: ${ }^{19}$

The conclusion that BSE would behave in the same way as scrapie was essentially a matter of judgement in the face of uncertainties, or an 'educated guess'. This was not something that the Working Party sought to conceal, as is clear from private correspondence which Sir Richard had with a number of scientists about BSE. In a letter to a Mr J Granger (a veterinary surgeon) on 25 October 1988, Sir Richard wrote: I have to say that it is my view that the risk of transmission of BSE to man is relatively small, but this view is based entirely on drawing a parallel with scrapie...(BSE Inquiry Report, Volume 4: 47).

The analogical argument ${ }^{20}$ that was central to the reasoning of the Southwood Working Party can be reconstructed as follows: 
BSE is similar to scrapie in certain respects.

Scrapie has not transmitted to humans.

$\therefore$ BSE will not transmit to humans.

Of course, we now know that the conclusions of these ignorance and analogical arguments were erroneous. BSE did indeed transmit to humans and cause CJD, a fact that was acknowledged for the first time on 20 March 1996 when Mr Stephen Dorrell, the then Secretary of State for Health, announced the emergence of new variant CJD to British Parliament. That these conclusions were ultimately shown to be erroneous did nothing to invalidate the initial legitimacy of the presumptive reasoning strategies that led to these conclusions. The ultimate defeat of specific presumptive conclusions in inquiry is in no way remarkable. Presumption is, after all, a highly tentative epistemic category that should be rejected as soon as countervailing conditions emerge. ${ }^{21}$ Moreover, the failure of one presumption in inquiry is not grounds for the rejection of presumptions in toto. The inherent defeasibility of presumptions is what makes this epistemic concept able to accommodate scientific error and to respond to such error in a way that does not involve the wider destruction of scientific inquiry. This is evident in the piecemeal fashion in which presumptive conclusions were rejected during the BSE inquiry as evidence from other sources became available. In this way, analogies with scrapie were used to guide decision-making on a whole range of issues beyond the question of the transmission of BSE to humans. One such issue was the cattle tissues to include in the Specified Bovine Offal (SBO) ban of November 1989: 
Tissues from cattle aged under six months were exempt from the ban on the basis that scrapie infectivity had not been found in lambs of this age (BSE Inquiry Report, Volume 1: 14).

The decision to exclude tissues from cattle in the first six months of life from the SBO ban was reversed in June 1994 when it became apparent that the pathogenesis of BSE was dissimilar in significant respects from that of scrapie (BSE Inquiry Report, Volume 1: 137-138). Specifically, a pathogenesis study that had been initiated at the Central Veterinary Laboratory (CVL) in December 1991 revealed infectivity in bovine tissues (e.g. ileum) earlier than had been expected using the scrapie model (BSE Inquiry Report, Volume 2: 121). The rejection of the presumption that it was safe to exclude the tissues of calves from the SBO ban displayed all the hallmarks of defeasible presumptions - the tentative epistemic commitment to this presumption was eroded as soon as scientists obtained evidence which led them to question the plausibility of this presumption. In fact, so immediate was the rejection of this presumption and any decisions ${ }^{22}$ that were based on it that the public inquiry team which investigated the handling of the BSE crisis described the response as 'a model of how government ought to handle such an issue' (BSE Inquiry Report, Volume 1: 138). At the same time, however, the defeat of the presumption that calf tissues were not infective did not trigger the wholesale rejection of every presumption that had been established through analogical reasoning based on scrapie. In particular, the decision to give special consideration to the safety of bovine tissues in medicinal products was premised on earlier scrapie research which had revealed that the parenteral route of transmission was more efficient than the oral route. ${ }^{23}$ 
If every presumption that had been licensed on the basis of an analogy with scrapie had been rejected once it was discovered that calf tissues were infective, the measures that were put in place to safeguard medicines and surgical products may well have been differently implemented, if they were implemented at all. ${ }^{24}$ Put quite simply, the demonstration of error in the case of one presumptive conclusion - the presumption that calf tissues were not infective - had a rather circumscribed impact on scientific inquiry into BSE. Presumption was the mechanism whereby this error could be absorbed without necessitating any wider destruction of the scientific inquiry into BSE. We will now consider how presumption also functioned in the scientific inquiry into BSE by licensing decisions in the practical sphere in the absence of complete and certain knowledge.

\subsection{Scientific Uncertainty and the Informal Fallacies}

If informal fallacies are particularly well adapted to dealing with the occurrence of error during scientific inquiry, they are even more suited to the task of bridging knowledge gaps during inquiry. These knowledge gaps were painfully evident to everyone involved in the BSE crisis, as can be seen from Mr Cruickshank's comments in section 1 above. It became clear to those whose task it was to contain the spread of the disease in cattle and to prevent its transmission to humans that key decisions would have to be taken against a wider epistemic context of uncertainty. The only other possible course of action delaying the institution of disease containment measures until a complete knowledge base became available - presented an unacceptable level of risk to the health of the population. It is against this background of uncertainty, I contend, that certain informal fallacies served to advance the inquiry into BSE. Specifically, the presumptive nature of these 
fallacies licensed decisions of the practical sphere and suggested important research directions when relevant knowledge was essentially lacking. In this section, we consider two such cases in which this occurred. A more detailed treatment of each case can be found in Cummings (2002).

We have already seen how the argument from ignorance formed the mainstay of scientific and ministerial pronouncements about the (lack of) risk of BSE to human health. Time and time again, consumers were told that that there was no evidence that BSE in cattle caused CJD in humans. The implication of this particular statement was, of course, that BSE did not cause CJD in humans. Of course, we now know that BSE did transmit to humans. Yet, this fact does nothing to diminish the very important role that this particular argument played during the BSE crisis. To understand this role, it is necessary to consider the type of knowledge that was required to address the question of transmission and whether there was a realistic prospect of scientists obtaining that knowledge in the short and (foreseeable) longer term. It was known, for example, that transmissible spongiform encephalopthies had very long incubation periods. ${ }^{25}$ On the assumption that BSE would exhibit an equally lengthy incubation period in humans, scientists were facing a situation in which they would be unable to address the question of transmission for many years to come. If disease containment measures were not established until BSE-related disease first emerged in humans, the number of people who could have become infected in the intervening years was potentially very large indeed. Ethical considerations prohibited the type of experimentation that would have directly addressed this question. Moreover, the closest knowledge of the transmission of 
spongiform encephalopathies to humans involved cases that either failed to replicate the species barrier that BSE would have to cross in order to infect humans ${ }^{26}$ or failed to replicate the oral route of transmission by means of which BSE would make its way into the human population. ${ }^{27}$ The knowledge base that was needed to address the question of the transmission of BSE to humans was thus incomplete in all key respects.

Unable to address the question of BSE transmission to humans directly, scientists had little option but to set this question to one side during inquiry. The argument from ignorance enabled scientists to disengage from the question of BSE transmission to humans and to address other questions for which there was at least a reasonable prospect of obtaining answers. By representing the question of transmission to humans as disconfirmed (this is, in effect, what the ignorance argument presented in section 2.1 achieved), there was no longer a requirement on scientists to actively pursue a response to it. If the question had remained unconfirmed, ${ }^{28}$ it would have continued to be subject to deliberation by BSE scientists during inquiry. The argument from ignorance had the beneficial effect of creating an economical order in which questions were examined during the BSE inquiry - where a question was unlikely to be addressed in either the short or longer term, it was set to one side to make way for questions that could be directly tackled. Quite apart from dismissing the important question of transmission to humans, BSE scientists were responding to the uncertainty that confronted them by instituting a rational epistemic policy. This policy enabled scientists to concentrate their cognitive and technical resources in areas were gains could reasonably be expected to be made. To this extent, the argument from ignorance served to facilitate inquiry under 
conditions that might otherwise have seen it grind to a halt. We will see shortly that this facilitative effect is the direct result of the presumptive character of this particular argument form.

The question of transmission of BSE to humans was also addressed through another argument form that was based upon an analogy with scrapie in sheep. Scientists argued that scrapie had not transmitted to humans in 250 years $^{29}$ and that BSE, which was presumed to be similar to scrapie (if not actual scrapie in cattle), would not transmit to humans either. In reality, the analogy with scrapie motivated a large number of research questions concerning BSE in addition to the question of transmission of the disease to humans. Just how many was evident in a paper produced in December 1988 by $\mathrm{Mr}$ Bradley of the CVL. The paper described five experiments that were already in progress at the CVL and which were addressing questions in the areas of epidemiology, clinicopathological studies, transmission, molecular biology and molecular genetics. All five experiments were premised on earlier research into scrapie in sheep. For example, molecular genetic studies were designed 'to determine any genetic factors involved in disease expression in cattle, and to determine whether an equivalent to the sinc or sip genes existed in cattle' (BSE Inquiry Report, Volume 2: 187). The sinc and sip genes are mice and sheep genes (respectively) that regulate the incubation period of natural and experimental scrapie. The list of tissues included in tissue infectivity studies was based on 'extrapolation of data from Hadlow's studies into scrapie in sheep' (BSE Inquiry Report, Volume 2: 194). By providing a research framework, the analogy with scrapie was able to generate many productive lines of inquiry for scientists to pursue when little 
was known about BSE. Analogical reasoning had the effect of advancing inquiry on grounds that held at least some degree of initial plausibility. It thus functioned to bridge gaps in scientific knowledge, gaps that would otherwise have adversely affected the progress of inquiry. This was possible at all because of the essentially presumptive character of the analogical arguments involved. We consider the role of presumptions in these arguments below.

The ignorance and analogical arguments that we have examined in this section were effective, I am arguing, in bridging gaps in scientific knowledge during the BSE crisis. Some examination of the nature of these arguments reveals why this was the case. Presumption was the operative epistemic concept in these different arguments. The concept of presumption exhibits a distinctly exploratory quality. It licenses investigators to pursue courses of action when knowledge is lacking. At the same time, it contains an implicit guarantee that should a particular course of action be found to be wanting, our tentative commitment to the presumption that warranted this course can be easily eroded. The 'epistemic damage' of running with a presumption that is ultimately shown to be incorrect is thus quite circumscribed. Certainly, it is sufficiently limited in extent not to deter an investigator, scientific or otherwise, from becoming committed to future presumptions or indeed to the same 'erroneous' presumption under different circumstances. The ignorance and analogical arguments that were used extensively during the BSE inquiry were presumptive reasoning strategies. These arguments conferred sufficient warrant on the presumptions that were generated by means of them that scientists were able to initiate research studies and implement disease containment 
measures. Yet, the merely tentative nature of these presumptions meant that when they began to appear problematic, scientists were readily able to reject them. Such rejection occurred in some cases in which it was warranted (e.g. the rejection of the presumption that it was safe to exclude calf tissues from the SBO ban). It is one of the tragedies of the BSE affair that it did not take place in every situation in which presumptions based on ignorance and analogical arguments were shown to be inadequate. We consider this issue in the next section.

\subsection{Scientific Dialectic and the Informal Fallacies}

Models of scientific inquiry that are based on disputation are by now well established. One of the most prominent proponents of this dialectical approach to scientific methodology is Nicholas Rescher. Rescher (1977) proposes a scientific dialectic in which:

[C]reative science appears in the light of an adversary procedure, with proponents and opponents carrying on a debate to secure the approbation of knowledgeable but “disinterested” (i.e., unaligned) parties - often as not the rising generation of specialists in the field (111; italics in original).

Within this dialectical approach, presumption and burden of proof is the mechanism by means of which evidential considerations in support of scientific theses are effectively managed. In this way, the opponent of a scientific thesis must advance grounds why a particular claim should not be allowed to stand. Where these grounds are judged to be 
sufficiently plausible, the opponent has successfully discharged his burden of proof. A presumption now exists in favour of his thesis, a presumption which the proponent (now opponent) must seek to dislodge through his own advancement of evidence. Where no evidence can be advanced against a thesis, or the evidence that is advanced is not judged to be sufficiently plausible, the thesis held as a presumption grows in epistemic stature. As the participants in inquiry take it in turn to discharge their burden of proof and secure a presumption in support of their thesis, the probative standing of theses is steadily revealed.

This dialectical model of scientific inquiry is only revealing of the grounds of theses to the extent that the participants in inquiry recognize when sufficient plausible evidence has been adduced to discharge a burden of proof. Where this burden of proof is unreasonably high and the corresponding presumption is excessively difficult to dislodge, the dialectical character of inquiry becomes distorted. Presumptions, which are typically defeasible, may become resistant to the type of countervailing conditions that would normally bring about their rejection. These presumptions can then persist in inquiry regardless of the plausibility of the evidence that is adduced against them. Many of the ignorance and analogical arguments that facilitated inquiry into BSE in the early stages of the epidemic became so deeply entrenched that the presumptions which they generated soon assumed the status of unassailable theses. Consider again the analogical argument based on scrapie that led scientists to believe that BSE would not transmit to humans. Even as evidence began to emerge, for example, that the host range of BSE was not the same as that of scrapie, scientists continued to subscribe to the view that BSE was bovine 
scrapie and that BSE was not transmissible to humans. Officials at the Department of Health and the Ministry of Agriculture, Fisheries and Food reported to ministers on 6 May 1990 that Bristol University had diagnosed a 'scrapie-like' spongiform encephalopathy in a domestic cat. It was known that scrapie had never been successfully transmitted by inoculation to a cat. So this was the first indication that BSE had a different host range from scrapie. If BSE could infect a cat, it was entirely possible that it could differ from scrapie in one other salient respect. This was the question of BSE transmission to humans.

So concerned were scientists and government ministers by this development that their immediate response was to attempt to downplay its significance for the question of transmission to an anxious public. Essentially, scientists and ministers continued to maintain that BSE was scrapie in cattle and, as such, BSE was unlikely to transmit to humans. However, in the light of this new evidence, scientists could only continue to adopt this position by adhering to a somewhat implausible claim. This was the claim that scrapie maybe had transmitted to a cat but that such transmission had gone undetected:

[I]f this could happen to a cat, why should not human beings suffer the same fate? Yet it was far too soon to jump to any such conclusion. It was possible that there had always been the occasional case of feline spongiform encephalopathy (FSE) which had gone unrecognised (BSE Inquiry Report, Volume 1: 128). 
This claim was implausible for two reasons. Firstly, the oral route of transmission was known to be less effective than transmission via inoculation. If inoculation had never transmitted scrapie to a cat, it was even less likely that transmission would have occurred orally. Secondly, scrapie had been in the sheep population for some 250 years. If cats were susceptible to scrapie, it is likely that natural transmission to cats would have been detected at some stage in this long history of the disease. While the impulse of scientists and government ministers to 'prop up' the original analogy of BSE to scrapie was an understandable one - ministers in particular were concerned to allay the public's fears that BSE would transmit to humans - this was only achieved at the expense of defending the non-transmission thesis against plausible counter-evidence, specifically the evidence that BSE and scrapie had different host ranges. The non-transmission thesis became a 'fossilized' presumption in the BSE inquiry in that it persisted in immutable form even as contrary evidence became available to scientists. What should have been a defeasible presumption came to be treated by scientists and government ministers alike as a well established fact. By failing to allow plausible counter-evidence to stand against the nontransmission thesis, scientists and government ministers had effectively subverted the dialectical mechanism of presumption and burden of proof in the BSE inquiry. While this subversion achieved the short-term goal of convincing the public that beef was safe to eat, it had adverse long-term consequences in terms of public distrust of scientific pronouncements about risk (Cummings 2005a).

\section{SUMMARY}


Presumption, it emerges, is a highly versatile epistemic concept. Its central characteristic of defeasibility means that presumption is ideally suited to the fallible nature of scientific inquiry. Even where scientific error does occur and specific presumptions fall by the wayside, the immediate effect on inquiry is rather limited. This is because our commitment to presumption is as easily broken as it is forged. As well as accommodating scientific error, presumption enables scientists to make decisions and pursue courses of action in the practical sphere when certain and complete knowledge is essentially lacking. Presumption thus has fundamental relevance to scientists who are confronted with newly emerging infectious diseases. These scientists must put into action disease containment measures when everything from type of pathogen to route(s) of transmission is unknown. It is in a context of epistemic uncertainty that the exploratory nature of presumption comes to the fore. This concept allows investigators to move forward in inquiry in the spirit that nothing ventured is nothing gained (or, perhaps more aptly, the scientist who does not venture forth in inquiry is likely to incur significant epistemic and practical penalties). Of course, as well as serving inquiry in these important ways, presumption can also subvert inquiry. Subversion occurs when the dialectical nature of this concept is neglected and plausible counter-evidence is not allowed to stand against presumptions. Such presumptions become 'fossilized' in inquiry and persist long after reasonable counter-evidence has been adduced against them.

All three aspects of presumption were manifested in the reasoning of scientists during the BSE crisis. That reasoning contained numerous examples of arguments that logicians have traditionally classified as fallacies. Two cases in point were the argument from 
ignorance and analogical argument. I have argued in this paper that these arguments were quite far from being fallacious; indeed, they conferred a number of epistemic gains on the inquiry of which they were a part. This was at all possible, I contended, because these arguments were presumptive in nature. Yet, the same presumptive forms of reasoning that served to advance inquiry under certain conditions - for example, when knowledge was lacking - stifled inquiry in other situations. This analysis of these argument forms represents a significant departure from traditional accounts of the fallacies. Firstly, the traditional distinction between fallacious and non-fallacious arguments is recast in terms of presumptions that are applied to the benefit of inquiry in some circumstances and are misapplied with adverse consequences for inquiry in other circumstances. Secondly, the fallaciousness of any argument becomes a context-sensitive affair and is related ultimately to the probative standards that operate in a particular setting. This study thus contributes to the 'pragmatic turn' that has occurred in fallacy theory in recent years (Cummings 2005b: 173). 


\section{NOTES}

1. Bovine spongiform encephalopathy (BSE) is a progressive, degenerative disease of the brain in cattle. Since its widespread emergence in the 1980s, many thousands of British cattle have developed the disease. In early 1993, BSE cases were being reported at a rate of around 1,000 a week (BSE Inquiry). BSE subclinical cases have now been linked to the development of a new spongiform encephalopathy in humans called new variant Creutzfeldt-Jakob disease (nvCJD).

2. It is arguable that containment was achieved more successfully in the case of SARS than in BSE. The outbreak of SARS in 2003 was contained in less than 4 months after its initial recognition. However, the outbreak affected 8,098 people, 774 of whom died as a result of the infection (Lingappa et al. 2004). The Pathology Department of the UK's Central Veterinary Laboratory first investigated the death of a cow that had developed BSE in September 1985 (BSE Inquiry, volume 1). Over 20 years later, cases of the disease are still being detected in cattle, although at much lower numbers than at the height of the epidemic (see note 1). For example, in 2007 there were 67 confirmed cases of BSE in the UK (Department for Environment, Food and Rural Affairs, 2008). Of course, given the lengthy incubation period of spongiform encephalopathies, the full extent of the human health toll of this disease is still unknown. The UK's National Creutzfeldt-Jakob Disease Surveillance Unit reports that between 1995 and March 2008, there had been 163 deaths caused by new variant CJD (a new form of CJD that is directly related to BSE in cattle). 
3. It is regrettable that the very scientific disciplines that need to examine how scientists make decisions under conditions of uncertainty often devote little or no effort to this important reflective exercise. Journals in epidemiology routinely do not publish the type of critical and philosophical discussions that this reflection requires. Indeed, the dearth of such discussions has prompted the development of a new, online journal. In this way, Phillips et al. (2004) state that 'the desire for new information means that the health science literature is overwhelmingly devoted to reporting new findings, leaving little opportunity to improve the quality of the science. Epidemiologic Perspectives \& Innovations was created to provide a forum for efforts to improve the quality of health science research and its applications'. Humanities and social scientific disciplines, including philosophy, sociology and politics, are very often the only fields in which scientific reasoning and decision-making come under any scrutiny.

4. Christakos et al. (2005: 47) describe the deductive and inductive reasoning rules that are integral to epidemic modelling. One inductive reasoning rule is the appeal to authority, a form of argument that has been frequently discussed in the literature on informal fallacies. These investigators also characterize a number of deductive and inductive fallacies. They include affirming the consequent and denying the antecedent as deductive fallacies, and simple generalization and causal generalization as inductive fallacies. 
5. Christakos et al. (2005: 22) believe that new forms of reasoning, which are not subsumed within formal logic, are integral to the efforts of public health scientists to respond to uncertainty: 'The intellectual process leading to the solution of an epidemic system should be viewed as the theoretical construct of epistemic cognition...the solution of such a system should follow certain rules of reasoning. But these rules are, in the final analysis, propositions about one’s epistemic cognition process...the epistemic cognition schema is more general than the logical one, because it involves representations and procedures (diagrams, visual images, mental metaphors, etc.) that may not be found in formal logic’.

6. 'And what we find in most cases, I think it should be admitted, is as debased, worn-out and dogmatic a treatment as could be imagined - incredibly tradition-bound, yet lacking in logic and historical sense alike, and almost without connection to anything else in modern logic at all. This is the part of his book in which a writer throws away logic and keeps his reader's attention, if at all, only by retailing traditional puns, anecdotes, and witless examples of his forbears' (Hamblin 1970: 12).

7. Or maybe not. As an anonymous reviewer of this paper pointed out, 'Aristotle, in the Topics, considers analogy exactly for the purpose that the author explains in this paper, e.g. advancing scientific knowledge. Indeed, at Topics 114b 25, for example, we find a topos that corresponds to analogy. What determines the fallaciousness of analogy is the degree of resemblance about the two things being compared'. 
8. Walton (1992) states that '[p]resumption is a speech act halfway between assertion and (mere) assumption or supposition in argument' (383).

9. '[W]hat will be evidentially sufficient in shifting a burden of proof hinges on the inherent seriousness of the contention at issue' (Rescher 2006: 17).

10. The precautionary principle was developed in the field of environmental health. Martuzzi and Bertollini (2004: 44) describe key aspects of this principle, one of which links it very directly to the operation of burden of proof: 'it suggests that the burden of proof might be reversed, from "recipients" to prove that an agent or technology is harmful to "proponents", to prove that it is innocuous'.

11. The expression 'retrospective validation' is taken from Rescher (1977) who aptly describes the epistemic gains that are made by presumptions during inquiry: 'In the first instance...presumptions have a merely provisional and regulative standing, though in the final instance they attain a suitable degree of factual-constitutive substantiation' (1977: 56; italics in original). The process by means of which presumptions are 'upgraded' is one of retrospective validation between the putative truths that are the output of inquiry and the presumptions that act as input: 'The logical structure of this justificatory process incorporates a feedback loop leading from the truths validated by the inquiry procedure back to the initial "merely presumptive" truths, so that the appropriateness of the initial, tentative, merely plausible presumptions can be reassessed. This points towards a cyclic process of revalidation and cognitive upgrading in the course of which presumptive 
theses used as inputs for the inquiry procedure come to acquire by gradual stages an enhanced epistemic status’ (Rescher 1977: 56-57; italics added).

12. Mill states that '[f]or every property... in facts, or in our mode of considering facts, which leads us to believe that they are habitually conjoined when they are not, or that they are not when in reality they are, there is a corresponding kind of Fallacy; and an enumeration of Fallacies would consist in a specification of those properties in facts, and those peculiarities in our mode of considering them, which give rise to this erroneous opinion (System, Book III, Chapter II, Classification of Fallacies, § 1; italics added).

13. Not only are errors persistent, but they also occur in abundance. In a study of the logic of error, Gabbay and Woods (to appear) propose an error abundance thesis in which 'beings like us make errors, lots of them'.

14. The eighteen fallacies include ad baculum, ad hominem, ad misericordiam, ad populum, ad verecundiam, affirming the consequent, denying the antecedent, amphiboly, begging the question, biased statistics, complex question, composition and division, faulty analogy, equivocation, hasty generalization, secundum quid, gambler's and ignoratio elenchi.

15. The terms of reference of the public inquiry were ' $[\mathrm{t}] \mathrm{o}$ establish and review the history of the emergence and identification of BSE and variant CJD in the United Kingdom, and of the action taken in response to it up to 20 March 1996; to reach 
conclusions on the adequacy of that response, taking into account the state of knowledge at the time; and to report on these matters to the Minister of Agriculture, Fisheries and Food, the Secretary of State for Health and the Secretaries of State for Scotland, Wales and Northern Ireland' (BSE Inquiry Report, Volume 1: xvii). The 16-volume report that was produced by the inquiry team is easily the most comprehensive account of the BSE crisis that is available. As such, it will provide the factual background to the discussion in this paper.

16. SEAC was established to advise the Ministry of Agriculture, Fisheries and Food and the Department of Health on matters relating to spongiform encephalopathies. It consisted of scientists who had considerable expertise in different aspects of human and animal health. Its members were Dr Tyrrell (Director of the Medical Research Council Common Cold Unit, Chairman of SEAC), Dr Will (Director of the CJD Surveillance Unit), Dr Watson (Director of the Central Veterinary Laboratory (CVL), 1986-1990), Dr Kimberlin (independent consultant in transmissible spongiform encephalopthies), Professor Fred Brown (a virologist), Professor Ingrid Allen (a neuropathologist), Professor Richard Barlow (pathologist and veterinarian), Mr David Pepper (veterinary surgeon), Dr William Hueston (epidemiologist), Professor John Pattison (a medical microbiologist), Professor John Collinge (Head of the Neurogenetics Unit at St Mary's Imperial College School of Medicine), Dr Michael Painter (a consultant in communicable disease control), Professor Peter Smith (human epidemiologist and statistician), Professor Jeff Almond (virologist and immunologist) and Mr Ray Bradley (Head of Pathology 
Department at the CVL between 1983 and 1995 and coordinator of BSE Research at CVL between 1987 and 1995).

17. Similar 'no evidence' claims were also repeatedly made by successive British government ministers who wished to allay public anxiety that BSE might be transmissible to humans. Their motivation for making these claims might well be explained by the fact that beef and dairy farming was a hugely profitable sector of agriculture in the UK. At the time BSE emerged, the output from milk, fattened cattle and calves totaled some $£ 5$ billion and comprised nearly 38 per cent of agricultural output in the UK (BSE Inquiry Report, Volume 1: 23).

18. Discussion of the argument from ignorance can be found in Schedler (1980), Walton (1992) and Walton (1996). Although individual characterizations vary, the argument has the following form: 'A is not proven true (false), therefore A may be presumed to be false (true)' (Walton 1992: 385). Applied to the BSE problem, the argument reads as follows: It is not proven true that BSE in cattle causes CJD in humans, therefore it may be presumed to be false that BSE in cattle causes CJD in humans.

19. The Southwood Working Party was established on the recommendation of Sir Donald Acheson (Chief Medical Officer, 1983-1991) 'to advise on the implications of Bovine Spongiform Encephalopathy and matters relating thereto’ (BSE Inquiry Report, Volume 4: 2). The Working Party consisted of experts in human and animal health. Its members were Sir Richard Southwood (Chairman of the Working Party and a professor of 
zoology), Anthony Epstein (a professor of virology), Sir John Walton (a clinical neurologist) and Dr William B. Martin (a veterinarian).

20. Walton (1995: 135) characterizes the argument from analogy as follows: 'Generally, case $C_{1}$ is similar to case $C_{2}$. A is true (false) in case $C_{1}$. Therefore $A$ is true (false) in case $\mathrm{C}_{2}$. Applied to the BSE problem, the argument reads as follows: Scrapie in sheep is similar to BSE in cattle. Transmission to humans has not occurred in the case of scrapie. Therefore, transmission to humans will not occur in the case of BSE.

21. Rescher (1977: 57) remarks that '[a]n initial presumption may well drop by the wayside in the long run. It is only normal and to be expected that this should happen, given the merely tentative probative nature of presumptions'.

22. As soon as health officials became aware of the findings of the pathogenesis study, an 'exceptional meeting' was called on 25 June 1994. The Chief Medical Officer, Dr Calman, said that he would be advising ministers that the distal ileum and thymus of calves should be proscribed as SBO. The Ministry of Agriculture, Fisheries and Food immediately wrote to the operators of all slaughterhouses, telling them of the proposed extension of the SBO ban (BSE Inquiry Report, Volume 1: 137-138).

23. The parenteral route of transmission describes the introduction of infection into the body via some route other than the mouth or bowel. Bovine-based products injected intramuscularly were a possible parenteral route of BSE transmission to humans. Earlier 
scrapie research indicated that the oral route of transmission was considerably less efficient than the parenteral route (Kimberlin and Walker 1989). Also, scrapie research that examined different parenteral routes found them to vary in terms of their efficiency of transmission (Kimberlin and Walker 1978). Intracerebral and intraspinal routes were generally the most efficient, followed by intravenous, intraperitoneal and subcutaneous routes.

24. The Committee on the Safety of Medicines issued guidelines to the manufacturers of pharmaceuticals on 9/10 March 1989. The public inquiry team described these guidelines as 'the single most important step taken to secure the safety of medicines' (BSE Inquiry Report, Volume 1: 177). The guidelines applied to all licensed products for injection, application to the eye or to open wounds. They required that 'no brain, neural tissue, thymus or other lymphoid tissue, placental tissue or cell cultures of bovine material should be used in manufacture' (Volume 1: 177).

25. Iatrogenic transmission of CJD (transmission through some form of medical treatment or surgery) has permitted the calculation of specific incubation periods for this disease. 'Central inoculation through neurosurgery, depth electrodes, corneal graft or dura mater graft results in disease after a mean incubation period of about 2 yr ... Peripheral inoculation through human pituitary derived growth hormone (hGH) or pituitary derived gonadotrophin $(\mathrm{hGnH})$ results in disease after an incubation period range from a minimum of $4 \mathrm{yr}$ to a maximum of at least $30 \mathrm{yr}$ with a mean of around 12 yr’ (Will 1993: 963). 
26. Prior to BSE, human-to-human transmission accounted for all cases of spongiform encephalopathies in individuals who did not develop sporadic CJD. Such transmission occurred via one of two routes: (1) surgical procedures that either used tissues that had been harvested from someone with CJD or that used equipment that had been previously used on someone incubating CJD and (2) the consumption of infected human brain matter as part of the practice of cannibalism in the Fore people of Papua New Guinea. This practice led to the development of kuru, a human transmissible spongiform encephalopathy (TSE) that was first described in the 1950s by Zigas and Gajdusek.

27. The only spongiform encephalopathy that has passed to humans via an oral route is kuru (see note 26). However, kuru is a human TSE and, as such, it did not have to cross the species barrier (unlike BSE). More commonly, parenteral routes of transmission have led to the development of spongiform encephalopathies in humans. In this way, CJD has transmitted to humans via corneal transplants, stereotactic intracerebral electrodes and human growth and gonadotrophin hormones derived from pooled pituitary glands from cadavers (BSE Inquiry Report, Volume 2: 25).

28. The argument from ignorance achieved the suppression of this possibility. This can be seen from the definition of ad ignorantiam advanced by Woods and Walton (1978), in which the argument is presented in terms of confirmation theory: 'the fallacy...consists in suppression of the possibility that $\mathrm{H}$ [the hypothesis] may be unconfirmed, i.e., the live possibility that there are no known data for $\mathrm{H}$ is omitted' (91; italics in original). 
29. 'Extensive retrospective studies together with a review of world literature led to the conclusion, published in 1987, that scrapie had never passed to humans despite opportunities to do so over the 250 years during which the disease had contaminated sheepmeat entering the human food chain’ (BSE Inquiry Report, Volume 2: 67). 


\section{Acknowledgements:}

The author wishes to acknowledge with gratitude the comments of two anonymous reviewers of this journal on an earlier version of this paper.

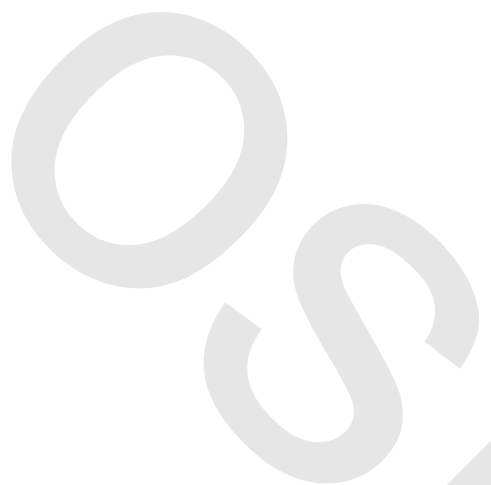




\section{BIBLIOGRAPHY}

Aristotle. 1955. On Sophistical Refutations. Translated by E.S. Forster \& D.J. Furley. London: William Heinemann Ltd.

BSE Inquiry. 2000. Volume 1: Findings and conclusions. London: Her Majesty's Stationery Office.

BSE Inquiry. 2000. Volume 2: Science. London: Her Majesty’s Stationery Office.

BSE Inquiry. 2000. Volume 4: The Southwood working party, 1988-89. London: Her Majesty’s Stationery Office.

Christakos, George, Ricardo A. Olea, Marc L. Serre, Hwa-Lung Yu, and Lin-Lin Wang. 2005. Interdisciplinary public health reasoning and epidemic modelling: The case of Black Death. Berlin and London: Springer.

Cummings, Louise. 2000. Petitio principii: The case for non-fallaciousness. Informal Logic 20: 1-18.

Cummings, Louise. 2002. Reasoning under uncertainty: The role of two informal fallacies in an emerging scientific inquiry. Informal Logic 22: 113-136. 
Cummings, Louise. 2004. Analogical reasoning as a tool of epidemiological investigation. Argumentation 18: 427-444.

Cummings, Louise. 2005. Giving science a bad name: Politically and commercially motivated fallacies in BSE inquiry. Argumentation 19: 123-143.

Cummings, Louise. 2005b. Pragmatics: A multidisciplinary perspective. Edinburgh: Edinburgh University Press.

Department for Environment, Food and Rural Affairs (2008) January 2008: Monthly summary statistics. London: DEFRA.

Gabbay, Dov M. and John Woods. To appear. Seductions and shortcuts: Fallacies in the cognitive economy. North Holland: Elsevier.

Hamblin, Charles L. 1970. Fallacies. London: Methuen.

Kimberlin, Richard H. and C.A. Walker. 1978. Pathogenesis of mouse scrapie: Effect of route of inoculation on infectivity titres and dose-response curves. Journal of Comparative Pathology 88: 39-47.

Kimberlin, Richard H. and C.A. Walker. 1989. Pathogenesis of scrapie in mice after intragastric infection. Virus Research 12: 213-220. 
Lingappa, Jairam R., L. Clifford McDonald, Patricia Simone, and Umesh D. Parashar. 2004. Wresting SARS from uncertainty. Emerging Infectious Diseases 10: 167-170.

Martuzzi, Marco and Roberto Bertollini. 2004. The precautionary principle, science and human health protection. International Journal of Occupational Medicine and Environmental Health 17: 43-46.

Mill, John Stuart. 1843. A system of logic, ratiocinative and inductive, being a connected view of the principles of evidence and the methods of scientific investigation. 2 volumes. London: Parker.

National Creutzfeldt-Jakob Disease Surveillance Unit. 2008. CJD statistics. http://www.cjd.ed.ac.uk/figures.htm. Accessed 26 March 2008.

Phillips, Carl V., Karen J. Goodman, Charles Poole, and Editors. 2004. Lead editorial: The need for greater perspective and innovation in epidemiology. Epidemiologic Perspectives \& Innovations. doi: 10.1186/1742-5573-1-1

Rescher, Nicholas. 1977. Dialectics: A controversy-oriented approach to the theory of knowledge. Albany: State University of New York Press. 
Rescher, Nicholas. 2006. Presumption and the practices of tentative cognition. Cambridge and New York: Cambridge University Press.

Schedler, George. 1980. The argument from ignorance. International Logic Review 11: 66-71.

Sidgwick, Alfred. 1884. Fallacies. New York: Appleton.

Walton, Douglas N. 1985. Are circular arguments necessarily vicious? American Philosophical Quarterly 22: 263-274.

Walton, Douglas N. 1992. Nonfallacious arguments from ignorance. American Philosophical Quarterly 29: 381-387.

Walton, Douglas N. 1995. A pragmatic theory of fallacy. Tuscaloosa, AL: The University of Alabama Press.

Walton, Douglas N. 1996. Arguments from ignorance. University Park, PA: The Pennsylvania State University Press.

Whately, Richard. 1836. Elements of logic. New York: William Jackson. 
Will, Robert G. 1993. Epidemiology of Creutzfeldt-Jakob disease. British Medical Bulletin 49: 960-970.

Woods, John. 1995. Appeal to force. In Fallacies: Classical and contemporary readings, eds. Hans V. Hansen \& Robert C. Pinto, 240-250. Pennsylvania: The Pennsylvania State University Press.

Woods, John. 2004. The death of argument: Fallacies in agent-based reasoning. Dordrecht: Kluwer Academic Publishers.

Woods, John and Douglas N. Walton. 1978. The fallacy of ‘ad ignorantiam’. Dialectica 32: 87-99. 